International Journal of Biomedicine | June 2021 - Volume 11, Issue Suppl_1: Abstracts from the Third Russian International Conference "Cryo-electron microscopy 2021: achievements and prospects"

\author{
POSTER ABSTRACT PRESENTATIONS
}

SESSION TITLE: EM RESEARCH RELATED TO MEDICINE

DOI: 10.21103/JJBM.11.Suppl_1.P33

\title{
Abstract P-33: Structural Basis for the Growing Microtubule End Recognition by End Binding Proteins
}

\author{
Alena V. Korshunova \\ Center for Theoretical Problems of Physicochemical Pharmacology, \\ Russian Academy of Sciences, Moscow, Russia
}

Background: Eukaryotic end binding proteins (EBs) can follow the growing microtubule end. EBs play a crucial role in microtubule dynamic instability and promote simultaneously growth rate and catastrophe frequency. It makes EBlike proteins perspective drag targets for a wide number of diseases. But the molecular mechanism of tip tracking by EB-like proteins remains unknown. Studies of mutants have revealed that the conservative amino acid Q102 (numbering relative to the human EB1 protein) plays a key role in the recognition of the growing microtubule end. However, the 3D structure studies revealed that this amino acid has no bonds with tubulin. In this work, we performed structural and phylogenetic analysis of EBs proteins to identify a possible molecular mechanism behind the plus end tracking.

Methods: UCSF Chimera10 was used for structural analysis. Phylogenetic analysis was performed with MEGA $X$ software. 3D structures of EBs and microtubules with different states of GTP hydrolysis were used (pdb 3JAK, 3JAS, 3JAT, 3JAW, 3JAL, 3JAR, 6DPU, 6DPV, 6DPW).

Results: We have shown that two conservative amino acids (K100, E106) should play an important role in the recognition of the microtubule plus end in addition to Q102. It was concluded that these amino acids together form the plus-end «navigation site» of EBs. Analysis of possible interaction of the «navigation site» amino acids with microtubules in different conformational states suggested that the main mechanism of growing microtubule end recognition is not due to an affinity increase for a certain state of tubulin in microtubules at their end, but it due to a significant affinity decrease in other parts of the microtubule as a result of steric clashes. 
Conclusion: Thus, the results of the analysis suggested the possible molecular mechanism that provides the tip tracking by EB-like proteins and allowed us to identify the key amino acids of this mechanism.

Key Words: microtubule $\bullet$ phylogenic analysis $\bullet$ structure.

This work was supported by the Russian Foundation for Basic Research (Grant No. 20-34-70159)

*Corresponding author: Alena Korshunova. E-mail: korshunova.av@gmail.com

International Journal of Biomedicine. 2021;11 Suppl 1: S26.

doi: 10.21103/IJBM.11.Suppl_1.P33

(C)2021 International Medical Research and Development Corporation 